\title{
Ueber das specifische Gewicht von Dämpfen bei sehr hohen Temperaturen;
}

von H. Sainte-Claire Deville und L. Troost*).

Die Bestimmung des specifischen Gewichtes von Dämpfen schwerflüchliger Körper ist für die Hülfsmiltel, welche die Physik bis jelzt bot, fast unmöglich; doch ist sie von grofsem Interesse für die Chemiker, welchen sie Beweise dafür abgeben könnte, dafs gewisse allgemeine Gesetze für alle Temperaturen als geltend angesehen werden.können. Die höchsten Temperaturen, für welche man das spec. Gewicht von Dämpfen bestimmt hat, übersteigen nicht viel $500^{\circ}$; solche Temperaturen wurden in den Untersuchungen von Dumas und Mitscherlich angewendet $*$ ). Es ist uns, nach sehr vielen Versuchen, gelungen, die drei grofsen Schwierigkeiten, welche unsere Vorgänger hinderten für höhere Temperaturen Bestimmungen auszuführen, zu überwinden; diese Schwierigkeiten liegen in der Wahl des Materials der Gefärse, in der Erzielung einer constanten Temperatur für die Dauer des Versuches, und endlich in der Bestimmung der Temperatur selbst.

*) Compt. rend. XLIX, 239.

*) In Malaguti's Traite de chimie findet sich die Angabe, dafs B in eau das spec. Gewicht des Schwefeldampfes bei $1000^{\circ}=2,218$ gefunden. Wir haben fast alle wissenschaftlichen Zeitschriften und das genanute Buch selbst durchsucht, um dic Beschroibung der von Bincau angewendeten Apparate und Vorfahrungsweisen kemmen zu lernen, doch ohne Erfolg. Wir müssen es somit aufrichtig bedatern, weiter Nichts über eine Untersuchung sagen zu können, deren Genauigkeit durch den Namen des Urhebers verbürgt wird; wir sind selbst auf die Vermuthung gekommen, Bineau möge nur aus den für niedrigere Temperaturen gefundenen Resultaten die richtige Zahl 2,218 für $1000^{\circ}$ gefolgert haben. 
Die Gefäfse, deren wir uns bedienen, bestehen aus Porcellan, und sie haben die Form eines Ballons von $280 \mathrm{CC}$. Inhalt und mit engern Hals; Herr Gosse, von Bayeux, hat sie uns mit grofser Zuvorkommenheit in der sorgfältigsten Weise anfertigen lassen. Ein solcher Ballon lälst sich in unvollkommener Weise miltelst eines kleinen, aus Porcellan bestehenden Cylinders von 1 bis $2^{\mathrm{mm}}$ Durchmesser verschliefsen, welcher sich in den engen Hals des Ballons fest einstecken läfst. Am Schlufs des Versuches bringt man das hervorstehende Ende dieses Cylinders mittelst des Knallgasgebläses zum Schmelzen, wodurch er an das Ende des Halses anschmilzt und dieser vollständig und luftdicht abgeschlossen wird.

Der Porcellanballon wird in einem eisernen Destillationsgefäfs erhilzt, dessen Einrichtung wir schon früher *) beschrieben haben und in welchem man miltelst Metalldämpfen eine constante Temperatur hervorbringt, gerade so wie wenn man einen abgeschlossenen Raum miltelst Wasserdämpfen auf $100^{\circ}$ erhitzen wollte, oder wie wir früher**) miltelst der Dämpfe aus siedendem Quecksilber oder siedendem Schwefel die constanten Temperaturen $350^{\circ}$ oder $440^{\circ}$ erhielten. Bei den Versuchen, deren Resultate wir jetzt mittheilen, wendeten wir die Dämpfe aus siedendem Cadmium $\left(860^{\circ}\right)$ oder siedendem Zink $\left(1040^{\circ}\right)$ an. Wir haben in dieser Weise ganz constante Temperaturen erhalten, wie wir uns durch die empfindlichsten Hülfsmittel überzeugen konnten.

Wir haben uns davon, die Temperaturen ganz scharf zu bestimmen, in der Weise frei gemacht, dafs wir immer mit Gefälsen aus derselben Substanz und von demselben Inhalt

*) Vgl. diese Annalen CV, 213.

**) Vgl, daselbst. 
operirten, in welchen wir nacheinander Joddampf *) und dann den Dampf des zu untersuchenden Körpers enthalten sein lielsen. Wir erhalten auf diese Art mit grolser Schärfe das Verhältnils der spec. Gewichte dieser beiden Dämpfe, für deren einen, den Joddampf, das spec. Gewicht von unseren Vorgängern und von uns selbst mit grofser Genauigkeit festgestellt ist. Durch diesen Kunstgriff wird es ganz unnöthig gemacht, die Temperatur zu bestimmen.

Wir können in dieser kurzen Mittheilung weder unsere Apparate noch unsere Operationsmethoden beschreiben; wir müssen uns mit der Bemerkung begnügen, dafs wir möglichst bei Dumas' Verfahrungsweise stehen geblieben sind und nur da uns Abweichungen erlaubten, wo die besonderen Umslände, unter welchen unsere Versuche angestellt wurden, diese nothwendig machten.

Wir theilen hier einige der hauptsächlichsten Zahlenresultate unserer Versuche mit.

Schwefel. - Bei $860^{\circ} *$ ) ist das spec. Gewicht seines Dampfes schon $=2,2$; aber damit diese Zahl definitiv angenommen werden könne, mulste nachgewiesen werden, dals sie bei noch höherer Steigerung der Temperalur sich nicht mehr ändert ${ }^{* * *}$ ). Letzleres ist nun in der That der Fall;

*) Joddampf wondeten wir fïr diese Aut von thermometrischen Bestimmmigen an der Stelle von atmosphärischer Luft an, weil der erstere etwa $9 \mathrm{mal}$ dichter als dic letzterc ist und die Wägungsfehler somit weniger einflu[srcich sind.

* Diese Zahlen sind berechnet ans der scheinbaren Ausdehnung, welche die Inft oder der Joddanpf in deın Porcellangcfärse zeigt; das Volum des Ietzteren nimmt anch bei den höchsten Temperaturen kaum merklich zu.

莯*) Wir schliefsen aus den schönen Cntersuchungen von Cahours, dafs man die Bestimmung des spce. Gewichtes eines Dampfes nur dann als eine definitiv gitilige betrachten kamn, wenn zwei fïr hinreichend weit unter einander abstehende Temperaturen 
denn wir haben für $1040^{\circ}$ dieselbe Zahl gefunden, welche durch mehr als 12 übereinstimmende Versuche festgestellt ist. Man kann somit mit aller Sicherheit sagen, dafs 1 Aeq. Schwefel (16) denselben Raum im Dampfzustand erfült, wie 1 Aeq. Sauerstoff (8).

Selen. - Der Selendampf zeigt dieselben Anomalien wie der Schwefeldampf. Das spec. Gewicht des Selendampfes ist bei $860^{\circ}=8,2$, bei $1040^{\circ}$ nur $=6,37$; erst über 1200 oder $1400^{\circ}$ darf man hoffen es constant zu finden. Neue, auf andere Principien gegründele Apparate, die bis für die höchsten Temperaturen sich brauchen lassen und mit deren Construction wir eben beschäfligt sind, werden uns ohne Zweifel gestatten, zur Zahl 5,44 zu kommen, welche die Theorie und die Analogie des Selens mit dem Schwefel erwarten lälst.

Phosphor. - Das spec. Gewicht des Phosphordampfes ist bei $1040^{\circ}=4,5$ (es berechnet sich zu 4,4), entsprechend 1 Volum für das allgemein angenommene Aequivalentgewicht dieses Körpers.

ausgefïhrte Versuche ïbereinstimmende Resultate geben. Somit ist ein einzelner Versuch unzurcichend; d. h. unan kann das spec. Gewicht eines Dampfes nur dann als festgestellt betrachten, wenn es oberlialb der Temperatur bestimmt wurde, von welcher an der Dampf sich wie ein Gas ausdehnt und den Ausdehnungscoëfficienten 0,00367 besitzt. Nur dann sind die Zahlen vergleichbar und können sie zur Prüfung des Gay-L uss a c'schen Volumgesetzes angewendet werden. Wir müssen indessen hier zahlreicher Versuche erwähmen, wo sich allerdings ein constanter störender Umstand geltend machte, der für das Quecksilber eine sonderbare Ausnahme von dieser Regel darthut. Veranlafst, ftir den Augenblick unsere Versuche zu unterbrechen, wollen wir dieses, in der Folge sich viclleicht nicht bestätigcnde, Resultat hicr anführen, um uns zu entschuldigen, dafs wir für diesen so wichtigen Körpor hicr noch keine Zahlen gegeben haben. Wir behalten uns vor, diese Untersuchungen nächstens wieder aulzunehmen. 
Deville u. Troost, über das spec. Gewicht etc.

Cadmium. - Das spec. Gewicht des Dampfes wurde für $1040^{\circ}=3,94$ gefunden; für eine Condensation auf 2 Volume berechnet es sich zu 3,87.

Chlorammonium. - Für $1040^{\circ}$ wurde das spec. Gewicht des Dampfes $=1,01$ gefunden; eine Condensation auf $8 \mathrm{Vo-}$ lume entspräche 0,92 .

Bromaluminizm : Beobachtetes spec. Gewicht des Dampfes: 18,62; für eine Condensation auf 2 Volume berechnetes : 18,51.

Jodaluminium : Beobachtetes spec. Gewicht des Dampfes : 27,0; für eine Condensalion auf 2 Volume berechneles : 27,8.

Die spec. Gewichte der Dämpfe der beiden letzteren Substanzen sind abgeleitet aus Versuchen, die im Dampf von siedendem Schwefel angestellt wurden. Das Jodaluminium besilzt eine sonderbare Eigenschaft, aus welcher hervorgeht, dafs die zwei es zusammensetzenden Elemente nur durch sehr schwache Verwandtschaft zusammengehalten werden. Es schmilzt bei $125^{\circ}$ und siedet bei $350^{\circ}$. Bei der letzleren Temperatur verhält sich sein Dampf so, wie wenn er freies Aluminium in einem besonderen Zustande enthielte; er entzündet sich an der Luft in Berührung mit einem brennenden Körper, und giebt dabei Jod und Thonerde. Mit Sauerstoff in einem starken Gefüse gemengt, detonirt dieser Dampf lebhaft, wenn man einen electrischen Funken durchschlagen lälst oder bei Annäherung einer Kerzenflamme, ähnlich wie es ein Gemenge eines brennbaren Gases mit Sauerstoff thut. Es ist klar, dafs die Elemente des Jodaluminiums hier in diesen besonderen Zustand übergeführt sind, welcher für alle zusammengesetzten Körper bei der Einwirkung einer hinreichend hohen Temperatur eintritt und welchen der Eine von uns als das Zerfallen chemischer Verbindungen in der Wärme bezeichnet hat*).

*) Vgl. diese Annalen CV, 383 .

D. $R$. 